\section{Appendix}

NAME:

ADDRESS:

\section{PRESENT POST:}

A: Training:

1. How long have you been training as a senior registrar?

2. How many sessions do you work a week?
(a) Clinical
(b) Academic Training
(c) Research

3. Do you do any on-call duty?

4. Do you find it necessary to work over and above your official sessions?

Pressure of work

To fit in case conference, etc.

YES/NO

When is your training due to finish?

6. What is your opinion of your training so far?

7. Are you getting the same range of training opportunities as full-time colleagues?

(a) Clinical

(b) Academic

(c) Additional experiences, e.g. opportunity of attending courses, meetings, etc.

(d) Research

8. Can you get to meetings outside the region? e.g. Section Spring Scientific Meeting or other College Meetings?

9. Have you access to a good library?

10. What are the main problems which you have encountered?
11. How far do you have to travel?

(a) To your clinical base

(b) For your academic sessions

12. Have you applied yet for any consultant posts?

13. Have you done any of your senior registrar training full-time?

If so, for how long?

14. Do you intend continuing to work part-time as a consultant?

15. Have you had any career advice since starting on the scheme?

16. If you can not move, do you know of possible job vacancies?

17. If there are few prospects in your area, have you been advised to stick it out in your chosen speciality or have you been advised to change, e.g. to psychogeriatrics?

B: Please will you kindly give us some information about your family and domestic circumstances?

1. Number and ages of children:

2. Partner's occupation:

3. Is your partner likely to need to move to find a permanent post?

4. Have you any other domestic ties, e.g. elderly parent requiring care?

5. Since coming on the scheme have you had any maternity leave?

6. Have you had any health problems since you have been on the scheme?

Please let us know of any other points you think are relevant to the planning of part-time higher training in child and adolescent psychiatry.

\title{
Coordinating care \\ Homepack: The use of a computer in community care
}

\author{
D. N. Martindale, Project Coordinator; M. Ness, Nurse Project Officer, \\ Coordinating Care Project, Community Psychiatry Research Unit; T. H. TURNER, \\ Consultant Psychiatrist, Psychiatric Day Hospital; and J. WRIGHT, Community \\ Development Worker, Psychiatric Day Hospital, Hackney Hospital, London E9
}

The project described in this paper developed from a DHSS funded scheme which was part of the Government's 'Helping the Community to Care' initiative. This was aimed at meeting a deficiency in the community - the lack of a coordinated system of after care for people suffering from long-term mental illness. The Mental Health Division of the DHSS described it thus:
"... Research and development work had already shown that by providing support, e.g. by providing relatives and patients themselves with basic information about the nature of the disorder and sources of help, the relapse rate can be reduced and also the strain on the carers. ..."1 
The use of computers is identified by many as a depersonalising and cold bureaucratic way for dealing with people. However, if the care of these vulnerable patients is to succeed, shared as it must be between so many agencies, there has to be some attempt at coordination, and computers do seem to offer a possible answer. In this paper we describe an attempt to use a computer to focus on the immediate need these patients have for more information about themselves and the services that are available to help them.

We have adapted a computer program to build on an existing policy in the Psychiatric Day Hospital at Hackney Hospital of allocating each patient a key or link worker, someone who is special to the patient. The keyworker and the patient compile an information package about the patient directly on to the computer. Three copies of this pack are printed out, one for the patient, one for the GP and one for a trusted person nominated by the patient. Many patients depend on relatives or friends for assistance in the community and it is important that these people have relevant information.

\section{The patient's printed information pack}

The pack is set out in letter form and tells the patient that the hospital is trying to improve the information it gives to people who live at home but attend hospital from time to time.

The names of personnel in the hospital and in the community who are known to the patient are listed with addresses and telephone numbers. This includes the consultant psychiatrist, the key worker, other hospital doctors, the GP, nurses, social workers, etc. who the patient might need to contact. A set of emergency telepone numbers is also included in the pack.

A section is left for special advice in which a key worker can set out personalised information for the patient. This could include the times of future appointments and occupation or other therapy sessions. The plan is for these Homepacks to be easily changed when the patient is reviewed; this is usually every 6-8 weeks at the Day Hospital in Hackney.

Another section of the pack deals with a description of the drugs the patient is taking and their possible side-effects. This information has been set out in easily understood language by a consultant psychiatrist. It is hoped to be able to offer translations of this in other languages in the future. Patients are asked to make sure they understood how many tablets they need to take, and how often, and to talk to their doctor or key worker if they are worried or unsure about their medications. Also included in the Homepack is information that some people qualify for free prescriptions and that leaflets about this can be found in Post Offices or DHSS offices. The Day Hospital also keeps copies of these leaflets.
General information sheets are given out because it is realised that for patients to function effectively at home, they need more than a description of hospital and para-medical agencies. Thus a list of all local agency offices: the unemployment, housing, social security and social services' addresses and telephone numbers is given routinely.

One of the authors has been building up a list of all community facilities that patients could use, and this is also given to the patients. Although both these sets of information are general, the keyworker will underline specific items for the patient.

We plan to extend this type of information as there are three directories being put together at the moment. A Social Services working party has been asking social workers in various offices, hospitals and group homes, to give details of the services they provide. A personal therapy list is being prepared by a multi-disciplinary working party at the hospital, and a users' group wants a central data resource base that includes where to find day centres, luncheon clubs, drop-in centres, etc. It is hoped that relevant information from these directories can also be given to the patient when the Homepack is made up.

\section{The nominated trusted person's copy of Homepack}

This also comes in the form of a letter and explains to the person that he or she has been nominated as someone whom the patient feels he can trust, and who is able to offer help from time to time. The information is similar except for the patient's 'special advice'. Before the pack is handed to the nominated trusted person the patient and the keyworker sign the copy.

\section{The GP's copy}

The GP is told of the Day Hospital's intention to convey relevant information about patients who live at home and attend the hospital periodically. It tells them what treatment has been arranged, and also that versions of the information pack have been given to the patient and, where appropriate, to a friend or relative nominated by the patient.

The GP is told that the hospital plans to keep this information up to date, and that the intention is to reproduce it each time the patient is formally reviewed by the Day Hospital's review team.

\section{Compilation}

The computer program stores most of the material needed for these three packs to be printed out. The specific individual information can be assembled with the patient in under half an hour.

The patient's details need to be accurately typed in and checked. The hospital staff, the medication and the dosage, etc. are coded and the code is simply 
typed in; the computer provides the rest. For example, amitriptyline has the code AMI. When this is typed the full drug name is filled in and the 'plain English' advice automatically included.

Once the patient's Homepack is compiled, it is stored and when the patient comes up for review after 6-8 weeks the pack can be called up on to the screen for modification. Again, it is hoped the patient will be present whilst the alterations are made. Access to this computer information is guarded by a password for the keyworkers.

\section{Comments}

The primary function of the Homepack is to provide information, both specific and general, to patients and their care-givers. By participating in the makeup of the pack, patients are given a chance to take responsibility for themselves, and by nominating a trusted person they have an advocate who is known to hospital and community staff. This accords well with the principle of advocacy that is supported by MIND. ${ }^{2}$ It also recognises that this trusted person, previously excluded from medical care and decision making, may have knowledge and expertise to contribute. Channels of communication, previously closed through the need for confidentiality, can be opened to enable better support to be given to the patient.

Another aim of the pack is to lessen the patient's dependency on the hospital and GP, through the realisation that other sources of help exist outside the health service. It should also lighten the load of doctors in the hospital or surgery setting, by promoting alternatives to the usual proposal for a change of medication.

There is a body of opinion still suspicious of computers. It is a hard enough task to introduce changes to well-established practices, without the obstacle of people's fears about new technology. This has to be handled with sensitivity and patience so that staff and patient can use the technology to provide a better service. For instance, it may be unrealistic for every keyworker to learn the use of the computer in the patient's presence. The patient needs care and attention in coping with this new experience and if the keyworker is too preoccupied with the new technology, the therapeutic relationship may be harmed. It may be easier therefore if some clinical details are placed on the computer beforehand and the patient asked to check their accuracy.

Fortunately, staff have had a sense of achievement after mastering a task which seemed alien and awe inspiring at first. It has also been possible to adapt the original program as we proceed so that it has become 'tailor made' for our Day Hospital.

The success of the scheme will ultimately be measured by the willingness of hospital managers and staff to use the pack as a complement to their present service, and also by how the information is applied by the patient. It is intended to carry out a detailed evaluation some 3-6 months after the initial pack has been given out. Such research is necessary to ensure that the Homepack is flexible, helpful, and 'user-friendly'.

\section{References}

${ }^{1}$ Williamson, P. M. (1984) Mental Health Division, DHSS, in letter to Dr J. Reed, Director, Community Psychiatry Research Unit, 21 December 1984.

${ }^{2}$ MiND (1983) Common Concern. MIND's manifesto for a new Mental Health Service. London: MIND.

Further information can be obtained from the authors at Hackney Hospital (Telephone (01) 985-5555, extension 8030). 